\title{
Future of internet of things (IoT) in 5G wireless networks
}

\author{
Abhiruchi Passi ${ }^{1 *}$, Deepak Batra ${ }^{2}$ \\ ${ }^{1}$ Department of Electronics and Communication Engineering \\ Manav Rachna International University, Faridabad \\ ${ }^{2}$ Department of Electronics and Communication Engineering \\ Manav Rachna International University, Faridabad \\ *Corresponding author E-mail: abhiruchi.fet@mriu.edu.in
}

\begin{abstract}
Internet of Things or IoT has evolved and will play a major role in our lives in the near future. Though in the nascent stage, it conceptually means an interconnected network consisting of physical objects, wearables, machines, buildings, automobiles and a variety of other types of devices that contain embedded technology to communicate and sense or interact with their internal states or external environment. With the advent of next generation $5 \mathrm{G}$ wireless technology around 2020, a wave of globally connected digital society will come into existence. In this paper we will discuss about the benefits which will be provided to IoT with the onset of 5G wireless technology and the challenges which IoT will offer to 5G.
\end{abstract}

Keywords: Internet of things; wireless communication; $5 G$;sensors; connected devices

\section{Introduction}

Kevin Ashton coined the term "Internet of Things" (IoT) to describe a system where the Internet is connected to the physical world via ubiquitous sensors. The Internet of Things is a computing concept wherein physical objects of everyday use are connected to the internet and sensors gather data from these devices process the data and use the information for various purposes. The schematic of IoT is shown in Figure 1.

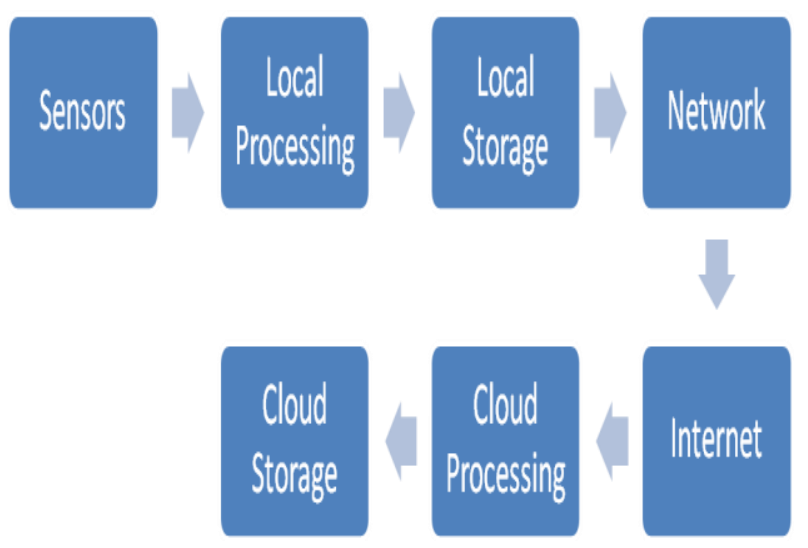

Fig. 1: Schematics of IoT
The sensors are small devices that have analog input and convert that input into digital or electronic data. The sensors consume less power and can be efficiently used for converting data gathered into such a format so that it can be easily sent through the Internet. Under local Processing and local Storage the data gathered from sensors is processed and is sent for computing. The Network and Internet is used for providing the required connectivity. The processed data is sent to cloud wherein the cloud aggregates and stores the data to generate inferences.

The application of Internet of Things (IoT) encompasses diversified fields as under:

1. Medical System: In the health care sector various devices are available which help in monitoring the health of the patient including his blood pressure, sugar level and heart rate using the IoT technology.

2. Transportation: With the use of Internet of things in transportation, parking has become smart. Also traffic light control, toll collection booths, road safety assistance system all have become automated thanks to Internet of Things.

3. Environmental Monitoring: Internet of things makes use of sensors which help in determining the quality of air, water and soil and also provide us information about the atmospheric changes. The IoT hardware covers a large geographic area thereby providing us data corresponding to the desired location.

4. Manufacturing: With the usage of Internet of Things in manufacturing sector, manufacturing of devices is taking place at a rapid rate and also optimization of products is taking place in real time thereby creating a dynamic response of the product in demand.

5. Energy management: Internet of things plays a major role in efficient management of energy. The electronic appliances that are making use of Internet of things help in saving energy and thereby minimize the energy consumption in offices as well as homes. 
6. Infrastructure management: Internet of things plays an important role in infrastructure projects like highways, bridges, and railway tracks, thereby making them automatic and eventually making them smart.

\section{Iot Requirements}

In order to understand the Internet of Things it is necessary to have knowledge about the associated hardware and software. The basic building block of IoT Hardware is shown in Figure 2.

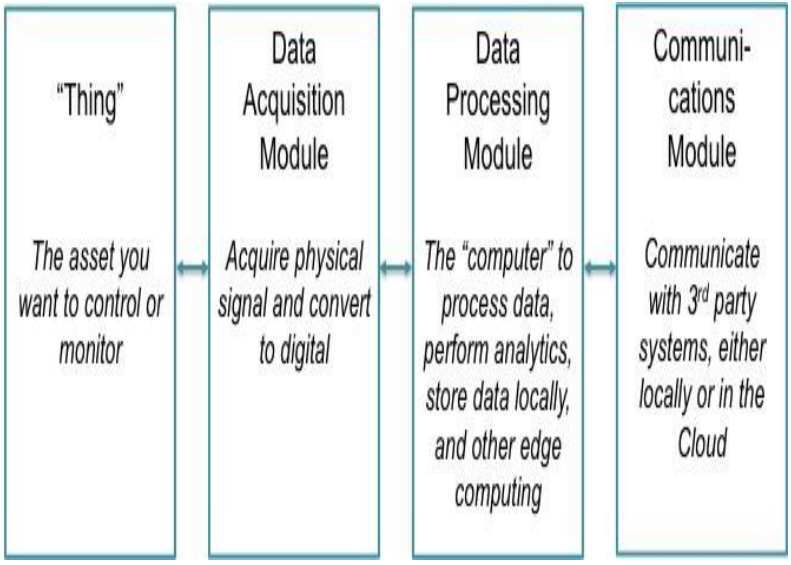

Fig. 2: Building Block of IoT Hardware

Thus, summarizing the components of IoT ,we can say that any physical object + (sensors, controllers and actuators $)+$ Internet $=$ Internet of Things. IoT software key areas include networking \& action through platforms and embedded systems. These individual and master applications are responsible for collecting data, integration of various devices, analytics of these devices in real time and process extension within the Internet of Things network. To sum up, Internet of Things requires billions of devices which are overloaded with embedded sensors and are required to be linked to the Internet, and should work or communicate at an enormous speed in an economical manner.

\section{Iot and 5G- Road Ahead}

With the commencement of Cellular systems (Analog Systems) in the early Eighties, Radio technologies recorded a fast and multi directional progress. Digital wireless communication systems are constantly meeting the growing requirements of society $(1 \mathrm{G}, 2 \mathrm{G}$, $3 \mathrm{G}, 4 \mathrm{G}$, and now $5 \mathrm{G})$.

Looking back, it is observed that every ten years, one generation is progressing in the area of wireless technology.

To begin with First Generation, $1 \mathrm{G}$ in the early eighties, followed by second Generation $2 \mathrm{G}$ in early nineties, Third Generation $3 \mathrm{G}$ in early twenty first century, fourth Generation 4G in 2010s and latest being fifth Generation 5G, the wireless technology is becoming more sophisticated and smarter.

\subsection{Introduction to $5 \mathrm{G}$}

From the time of introduction of $1 \mathrm{G}$ in 1982 , a novel generation of wireless communication is appearing around every decade. The primitive $1 \mathrm{G}$ service was succeeded by $2 \mathrm{G}$, which commenced text messaging features in 1992. Thereafter, 3G networks appeared in 2001, which allowed phones to access the Internet. Thereafter, in 2012, 4G emerged, which added video streaming features. Now, $5 \mathrm{G}$ is being developed with gigabit speeds. The first $5 \mathrm{G}$ networks are likely to be unveiled in 2020 .

$5^{\text {th }}$ Generation wireless Network or $5 \mathrm{G}$ is the impending revolution of mobile technology. The uses and special features are much above the anticipation of a normal person. With its super- high speed, it has the capability to change the sense of cell phone usability. Some of the features of $5 \mathrm{G}$ are shown in Figure 3

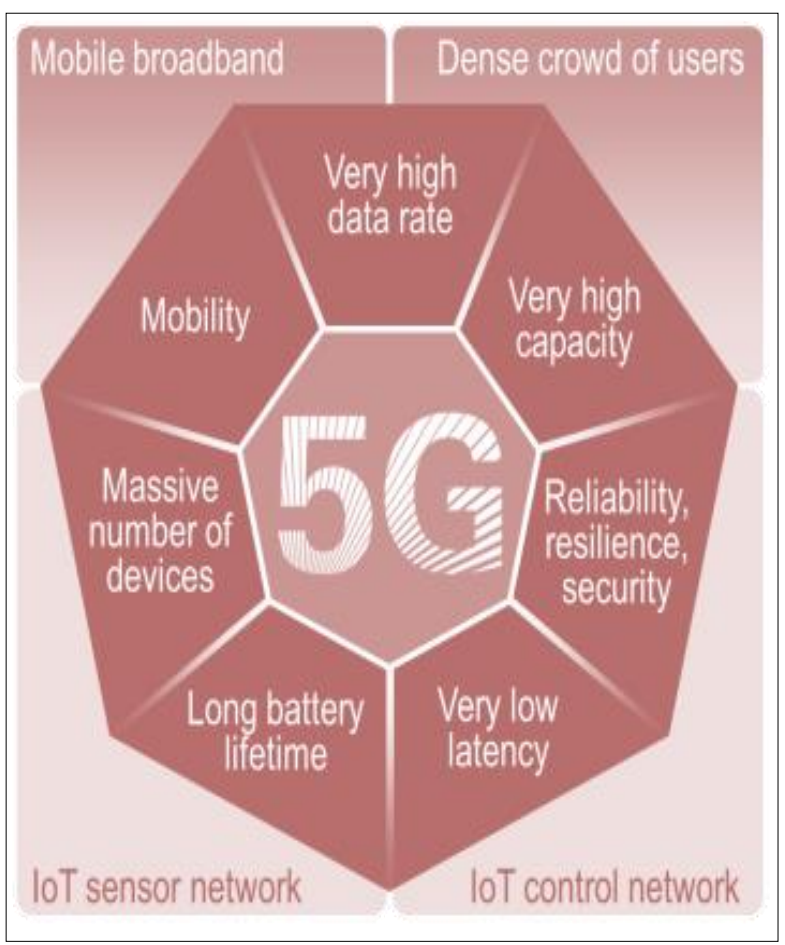

Fig. 3: Features of $5 \mathrm{G}$

As depicted in Figure 4, the model of system of 5G is purely IP based architecture which is designed for mobile as well as wireless networks. The system consists of a user terminal, followed by various independent as well as autonomous technologies for radio access. Each and every radio technology acts as the IP link for the Internet world. The main purpose of IP technology is to make sure that adequate control over data is possible for optimum routing of packets for application specific sessions from client applications to servers on the Internet.

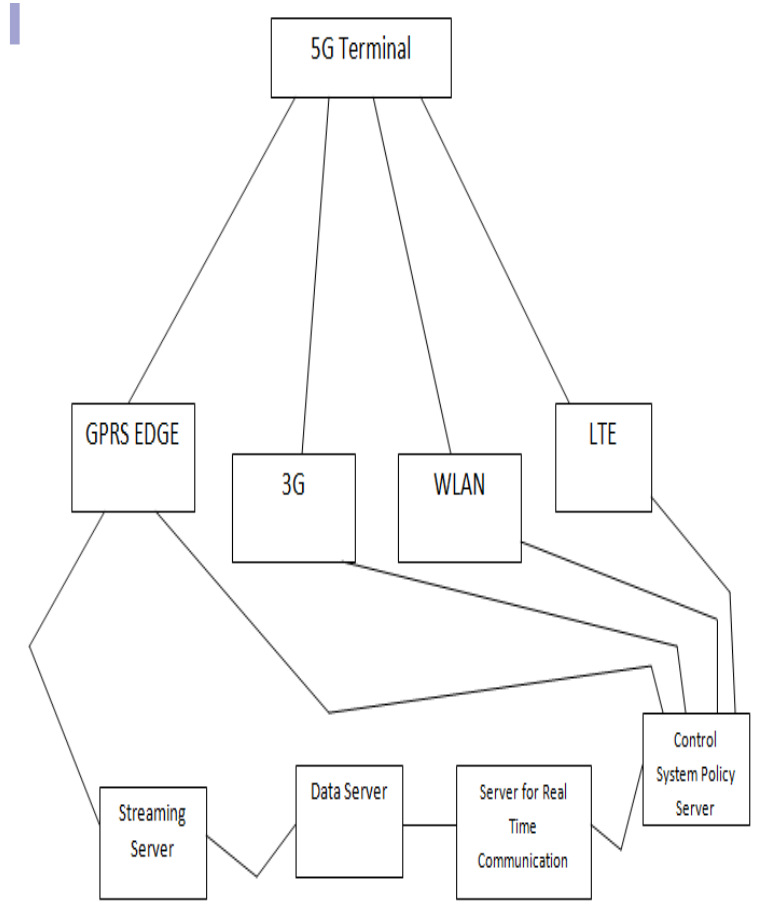

Fig. 4: Architecture of 5G 


\subsection{Advantages of $5 G$}

Few of the prominent advantages of $5 \mathrm{G}$ are as mentioned below:

- It will enable similar global standards for everyone.

- Network will easily be available for carrying out various processes.

- People will be able to use their laptops and similar types of devices (mobiles) anywhere as well as any time with high speed and good connectivity.

- The World will become a WiFi zone in real sense.

- The intelligent radio technology shall enable various versions of radio technologies to use or share the common spectrum with full efficacy.

- User will be able to get the radio signal even at greater altitudes.

\subsection{Advanced Features}

As compared to earlier radio technologies $5 \mathrm{G}$ has the following superior features:

- Almost capable to give ultra high speed ranging from 1 to 10 Gbps.

- Delay shall be 1 millisecond which will include round trip on end to end basis.

- Per unit area bandwidth will be 1000 times.

- It will be possible for many devices to inter connect.

- Enables global coverage.

- Usage of network energy will be reduced by $90 \%$

- Longer battery life.

The devices encompassing the Internet of Things are likely to increase at an exponential growth and it is assumed that the IoT installation will rise to 26 billion units by 2020 . However none of the existing network has the ability to cater to the requirement of such enormous number of devices. A whole novel network infrastructure shall be necessary to get the full benefit of IoT. Luckily $5 \mathrm{G}$, the forthcoming generation of wireless networks wil be able to support a large quantity of devices. The flexibility of supporting a vast variety of machine to machine communication is one of the basis of the $5 \mathrm{G}$ network.

$5 \mathrm{G}$ network will not only be able to cater to the requirement of applications requiring high bandwidth like streaming of high definition video, but it will also be able to support the ultra low latency which is required for applications which may include online gaming, remote surgery or even video conferencing. 5G architecture will also include a novel standard known as NB-IoT (Narrowband IoT) which will support enormous number of low bandwidth devices. This multi facet feature of $5 \mathrm{G}$ network will facilitate creation of seamless connectivity linking people as well as things. 5G network will also link cloud based resources for processing as well as storing of the enormous quantity of data generated by all the sensors.

\subsection{Enabling Factors of 5G for IoT}

$5 \mathrm{G}$ the future technology will facilitate expansion of Internet of things in the following ways:

- Higher bandwidth: Higher data rates as well as higher bandwidth facilitated millimeter wave technology. Researchers are in the process of designing $5 \mathrm{G}$ to support 1000 times the traffic that is being handled by the existing networks. This also gives speed of the range of $10 \mathrm{Gbps}$.

- Smaller device packaging: 5G shall be operated at frequencies upto $80 \mathrm{GHz}$ as compared to existing $4 \mathrm{~g}$ network supporting frequencies upto $6 \mathrm{GHz}$ thereby shrinking the size required for antennas upto $1-10 \mathrm{~mm}$ as compared to few centimeters for $4 \mathrm{G}$

- Millisecond latency: Latency may be defined as the time required to transmit one packet of data. The existing $4 \mathrm{G}$ networks provide latency as low as 25 miliseconds. 5G networks will provide latency less than 1 milisecond which is required for critical applications such as self driving vehicles as well as surgeries aided by robot, where any latency in transmission may mean death or life.

- High capacity networks: Networks with high effectiveness as well as efficacy, connecting billions of physical objects at lightning speed will be the key feature of 5G technology. The new era is expected to bring in novel services which completely leverage software defined networks (SDN's) as well as network function virtualization (LFV). These technologies facilitate building and configuration of networks centrally and automatically without any manual intervention at device level. NFV together with SDN uses network slicing as well as mobile computing to migrate functions of network to virtual machines.

- Forward compatibility: 5G technology will be forward compatible i.e. it will work with all the various types of devices to be connected in the future. Since newer devices will keep on adding in the IoT network, this is one of the key requirements to support the growth of IoT.

\subsection{Issues to be Addressed:}

(i) Some of the issues to be addressed for implementation of IoT in $5 \mathrm{G}$ networks are as under:

- Global reachability: The devices enabled, should be located and identified globally.

- Mobility support: The devices enabled should be connected even when mobile.

- Richer communication patterns: Various patterns of communication like anycast, publish/ subscribe, query/ resolve etc should be enabled.

- Resource efficiency: The requirement for network capacity, energy or computation should be minimized.

$5 \mathrm{G}$ will help to overcome the above challenges in the following ways:

- Millimeter Wave : 5G will work on waves which have very small length ranging from $1-10 \mathrm{~mm}$

- Small Cell: 5G technology will use base stations which are portable as well as small in size that requires bare minimum power for operation and will be placed every 250 meters approximately across the city.

- Massive MIMO: In 5G, base stations will be capable of receiving as well as sending signals from greater number of users simultaneously thereby increasing the mobile networks capacity. Beam forming and Full Duplex Technology also help in overcoming the involved challenges.

(ii) Security concerns: Since billions of devices will be part of IoT providing robust security solutions will be a major challenge. The IoT systems differ from traditional systems such as big data, enterprise application, cloud computing etc in the following ways:

- Uncontrolled Environment: Many physical objects will be part of uncontrolled environment wherein these objects will travel to unreliable surroundings and without supervision.

- Mobility: Continuous presence as well as stable network connectivity cannot be guaranteed in such an environment.

- Physical accessibility: Sensors in the IoT system may be accessible publicly. These include traffic control cameras as well as environmental sensors.

- Heterogeneity: IoT will be a heterogeneous system containing multiple things from various manufacturers. This will necessitate version compatibility as well as inter-operatibility.

- Scalability: The enormous amount of connected physical objects will require scalable products. This will affect the security mechanism as hierarchical public key infrastructure (PKI) and distributed methodologies like pair wise symmetric key exchange will not be able to scale with IoT.

- Constrained Resources: IoT will require lightweight solutions for security as resources are limited. There are energy limitations (Battery powered devices) and also computation restrictions (Micro 
sensors). So IoT things will not be able to handle complex computational cryptographic algorithms.

\section{Conclusion}

The Internet of Things is growing at a very rapid rate and will change the way things work. With vast areas of prospective applications like health care, shopping, smart manufacturing, Intelligent homes, Smart cities, wearables etc, IoT will surely play an important role in our day to day lives. The development of $5 \mathrm{G}$ networks is taking place at a very fast rate and is expected to be launched by 2020. The advantages and features of 5G are aptly suitable to support IoT in a big way. With $5 \mathrm{G}$ being hot topic of interest for researchers its implementation in the era of IOT will enhance the usage of Internet of Things technology in a huge way. As IoT will help make our life easy by automating the physical objects, the emergence of $5 \mathrm{G}$ will help IoT take a big leap.

\section{References}

[1] L. Atzori, A. Iera and G. Morabito, "The internet of things: A survey," Computer networks, pp. vol. 54 , no. 15 , pp. 2787-2805, 2010.

[2] Ericsson, "More than 50 Billion Connected Devices," 2011.

[3] S. Andreev, O. Galinina, A. Pyattaev, M. Gerasimenko, T. Tirronen, J. Torsner, J. Sachs, M. Dohler and Y. Koucheryavy, "Understanding the IoT connectivity landscape: a contemporary M2M radio technology roadmap," IEEE Communications Magazine, 2015.

[4] N. Mitton, S. Papavassiliou, A. Puliafito and K. S. Trivedi,

[5] "Combining Cloud and sensors in a smart city environment," EURASIP journal on Wireless Communications and Networking, 2012.

[6] M. Zorzi and e. al., "From today's intranet of things to a future internet of things: a wireless-and mobility-related view," IEEE Wireless Communications, 2010

[7] "3GPP TS 22.368, "Service Requirements for Machine-Type Communications (MTC)," V13.1.0,," Dec. 2014.

[8] A.Gupta and R. K. Jha, "A Survey of 5G Network: Architecture and Emerging Technologies," IEEE Access, 2015.

[9] https://5g.ieee.org

[10] S.V.Manikanthan and K.Baskaran "Low Cost VLSI Design Implementation of Sorting Network for ACSFD in Wireless Sensor Network", CiiT International Journal of Programmable Device Circuits and Systems,Print: ISSN 0974 - 973X \& Online: ISSN 0974 - 9624, Issue : November 2011, PDCS112011008.

[11] Rajesh, M., and J. M. Gnanasekar. \&quot;An optimized congestion control and error management system for OCCEM.\&quot; International Journal of Advanced Research in IT and Engineering 4.4 (2015): 1-10

[12] T. Padmapriya and V. Saminadan, "Improving Throughput for Downlink Multi user MIMO-LTE Advanced Networks using SINR approximation and Hierarchical CSI feedback", International Journal of Mobile Design Network and Innovation- Inderscience Publisher, ISSN : 1744-2850 vol. 6, no.1, pp. 14-23, May 2015.

[13] S.V.Manikanthan and D.Sugandhi "Interference Alignment Techniques For Mimo Multicell Based On Relay Interference Broadcast Channel "International Journal of Emerging Technology in Computer Science \& Electronics (IJETCSE) ISSN: 0976-1353 Volume- 7 ,Issue 1 -MARCH 2014 